\title{
El hábeas data: mecanismo de garantía procesal frente a las nuevas tecnologías
}

\author{
Habeas data: procedural guarantee mechanism against new \\ technologies
}

\author{
Luis Oswaldo Ordóñez Pineda \\ loordonez@utpl.edu.ec \\ Universidad Técnica Particular de Loja, Ecuador
}

Recibido: Abril 2018 / Revisado: Mayo 2018 / Aceptado: Junio 2018/ Publicado: Julio 2018

\begin{abstract}
RESUMEN
La garantía jurisdiccional de Hábeas Data constituye un mecanismo procesal que atribuye protección constitucional frente al impacto de las nuevas tecnologías de la información y comunicación (TIC), en lo que respecta a la tutela del derecho fundamental a la autodeterminación informativa. La metodología aplicada para el estudio de este fenómeno jurídico se derivó de la teoría tridimensional del derecho. Así, el objetivo de esta investigación se justificó a partir del estudio y análisis del régimen jurídico en Ecuador para la garantía del Habeas Data; y, caracterización de los presupuestos jurídicos necesarios para garantizar su ejercicio en la sociedad de la información. Al respecto, la doctrina considera que el habeas data y el derecho a la autodeterminación informativa constituye un instituto de garantía de otros derechos fundamentales y que, en la sociedad de la información, no se trata de impedir el uso de TIC sino de conciliar estas con el respeto de la dignidad del hombre. Como principales resultados están la importancia de las resoluciones de la Corte Constitucional en donde se precisa la incorporación en el Derecho de nuevos conceptos relacionados con esta garantía. Cardinalmente, la libertad informática y autodeterminación informativa se presentan como instituciones jurídicas orientadas a tutelar no solamente el "acceso" sino también el "control" sobre los datos personales. Por ello, en la era tecnológica se concluye que el Hábeas Data debe garantizar la libertad informática en lo concerniente al tratamiento "ilegítimo" y "no autorizado" de la información personal.
\end{abstract}

Palabras clave: Habeas data; Autodeterminación informativa; Datos personales; Derechos fundamentales; Garantías jurisdiccionales

\begin{abstract}
The jurisdictional guarantee of Habeas Data constitutes a procedural mechanism that attributes constitutional protection opposite the impact of new information and communication technologies (ICT), in what concerning the protection of the fundamental right to information self-determination. The methodology applied to the study of this legal phenomenon was derived from the three-dimensional theory of law. In this way, the objective of this research was justified with base on the study and analysis of the legal government in Ecuador for the guarantee of Habeas Data; and, characterization of the legal assumptions necessary to guarantee its exercise in the information society. In the case, the doctrine considers that habeas data and the right to informative self-determination constitute an institute for the guarantee of other fundamental rights and that, in the information society, the object is not to restrict the use of ICT, rather the intention is to reconcile them with respect for the dignity of citizens. The main results are the importance of the resolutions of the Constitutional Court where the incorporation into the Law of new concepts related to this guarantee is required. Fundamentally, informational freedom and informational self-determination are presented as legal institutions aimed at protecting not only the "access" but also the "control" of personal data. Therefore, in the technological era, it is concluded that the Habeas Data must guarantee the freedom of information concerning the "illegitimate" and "unauthorized" treatment of personal information.
\end{abstract}

Key words: Habeas data; Informative selfdetermination; Personal data; Fundamental rights; Jurisdictional guarantees. 


\section{INTRODUCCIÓN}

Los progresivos cambios en el marco regulador de la información personal han logrado instituir en la garantía jurisdiccional del habeas data varias denominaciones que pretender abarcar no solamente su fundamento sino también el conjunto de facultades que se atribuyen a las personas frente al tratamiento de su información.

Indudablemente, una de las principales fuentes de este cambio ha sido los avances tecnológicos y que exige del Derecho la articulación de nuevos preceptos elevados en el orden constitucional a libertades fundamentales y garantías constitucionales como instituciones reguladoras de hechos que afecte a la dignidad humana.

Considerándose con toda seguridad como la primera resolución sobre la materia, en 2001 el entonces Tribunal Constitucional en la Resolución Nro. 281 reconoció que la institucionalización de la garantía de habeas data en el Derecho Constitucional Latinoamericano ha ido progresivamente advirtiendo "nuevas dimensiones con la expansión de la informática, los sistemas de Internet y conjugando con aquellos derechos que, de modo directo o mediato sirven para tutelar o garantizar esos derechos inalienables y universales, como son aquellos ligados a la dignidad del ser humano".

Asimismo, posterior al reconocimiento del derecho fundamental a la protección de datos de carácter personal o de autodeterminación informativa en la Constitución de 2008, la Corte Constitucional en la Resolución Nro. $19^{2}$ ha señalado que el texto constitucional actual consagra al habeas data como "un derecho fundamental en sí

1 Véase el Registro Oficial Suplemento 281 de 9 de marzo del 2001 (caso signado con el Nro. 39-2000HD).

2 Véase el Registro Oficial 18 de 3 de septiembre del 2009 (caso signado con el Nro. 14-09-EP). mismo, independiente de otros y como un mecanismo de protección de otros derechos fundamentales".

Por tanto, tal como señala Davara (2005), el habeas data vinculado como un derecho fundamental se refiere:

...al amparo debido a los ciudadanos contra la posible utilización por terceros, en forma no autorizada, de sus datos personales susceptibles de tratamiento automatizado, para de esta forma, confeccionar una información que, identificable con él, afecte a su entorno personal, social o profesional, en los límites de su intimidad.

De esta manera, esta investigación tiene especial importancia a partir de que la garantía de habeas data se concreta con la protección de la información de carácter personal a través de la tutela del derecho a la autodeterminación informativa. Por ello, especial enfoque tiene el análisis del control y tratamiento de los datos personales mediante los derechos de acceso, rectificación, cancelación y oposición. Se concibe desde esta perspectiva que el habeas data -como un mecanismo de protección de otros derechos fundamentales- se constituye como una acción de control constitucional de un instituto de garantía de otros derechos fundamentales.

Como señala Murillo de la Cueva y Piñar (2011) el derecho a la autodeterminación informativa debe considerarse como un instituto de garantía por cuanto "siendo como es un derecho fundamental, es asimismo requisito para que otras libertades sean respetadas. Impide (debería impedir) que la información disponible sobre las personas pueda ser utilizada en contra de sus derechos y libertades" (p.109). Al respecto, de conformidad a la resolución que antecede, la Corte Constitucional en coincide con la doctrina en posicionar que este instituto de garantía protege entre otros derechos, "el 
derecho a la honra, al honor, a la intimidad, al buen nombre, a la imagen, a la verdad, al patrimonio, a la privacidad, a la voz y a la autodeterminación informativa frente al abuso y negligencia en el tratamiento de la información".

Bajo las anotaciones que anteceden, la hipótesis y objetivos que se pretenden desarrollar en la investigación surgen a partir del estudio el hábeas data como un mecanismo de garantía procesal frente a las nuevas tecnologías lo cual nos permitirá contextualizar el ejercicio de los derechos de acceso, rectificación, cancelación y oposición, tomando en cuenta que el desarrollo de las denominadas tecnologías de la información y comunicación también afecta al tratamiento de los datos de carácter personal como resultado de su circulación ilimitada y el libre acceso que se puede ejercer en una sociedad en red, evidentemente, sin mediar el consentimiento de las personas.

Asimismo, se pretender conceptualizar que el habeas data y el derecho fundamental a la autodeterminación informativa se configuran como un derecho fundamental en sí mismo que atribuye a la persona el derecho de controlar su información frente a posibles violaciones de los derechos de honra, intimidad, privacidad, etc.

En suma, a través de este estudio se tratará de afianzar la importancia del habeas data frente a las nuevas tecnologías. El debate teórico permitirá que en la práctica se materialice la efectiva tutela del derecho a la autodeterminación informativa y a su vez, procesalmente, se concrete la efectiva seguridad jurídica mediante las facultades que atribuye el habeas data. Se destacan en este contexto dos aspectos de singular importancia. El primero relacionado con el poder de controlar el tratamiento de la información e imponer a terceros limitaciones a través del derecho fundamental en la constitución y mediante la regulación en su ejercicio; y, el segundo, relacionado con el control constitucional consolidado en el habeas data.

\section{METODOLOGÍA}

La metodología de investigación se desprende de la teoría tridimensional del derecho. Es decir, se aborda el problema de investigación desde una perspectiva holística de tal suerte que el derecho fundamental a la protección de datos personales y el hábeas data como un mecanismo de garantía procesal frente a las nuevas tecnologías serán conceptualizado como hecho, valor y norma.

Con esta base y a fin de justificar los objetivos propuestos, la investigación desarrollada es de tipo: a) Explicativa, por cuanto consideró el estudio y análisis del régimen jurídico en Ecuador para la garantía del Habeas Data; y, b) Analítica, por cuanto se conceptualizó y caracterizó en la sociedad de la información los presupuestos jurídicos necesarios para garantizar su ejercicio en la sociedad de la información.

\section{RESULTADOS}

Según expone Pérez Luño (1996), el derecho a la protección de datos personales se fundamenta en:

Garantizar a los ciudadanos unas facultades de información, acceso y control de los datos que le conciernen. Pero esta forma de intimidad no se concibe como un valor intrasubjetivo, sino como autodeterminación del sujeto en el seno de sus relaciones con los demás ciudadanos y con el poder público (p.44).

Se evidenciaron dos aspectos de singular importancia en materia de tratamiento de la información de carácter personal; el primero que es el control de los datos personales y el segundo relacionado a la distinción de lo público y lo privado.

En relación al control, se estima que la protección de los datos personales debe ajustarse a ciertos principios que regulen el 
intercambio de datos e información de carácter personal y que estén encaminados a proteger los derechos personalísimos que se derivan del tratamiento de la información. La importancia en este contexto implica, también, poder determinar hasta qué punto se posee la facultad de compartir y utilizar información.

La regulación de la tipología de datos personales es bastante diversa por lo que se precisa la necesidad de establecer los límites frente al tratamiento de la información a efecto de garantizar en la práctica su efectivo cumplimiento en relación a otros derechos fundamentales.

En todo caso, Murillo de la Cueva y Piñar (2011) precisan también que:

...el desconocimiento no es algo exclusivo de los ciudadanos. Se extiende a instituciones públicas $\mathrm{y}$ privadas que disponen de amplios volúmenes de información personal $\mathrm{y}$, a veces, va acompañado por un escaso celo, cuando no despreocupación, en la aplicación de las normas legales y reglamentarias que la protegen.

Por tanto, se reafirma la preocupación acerca que en muchos ordenamientos jurídicos existe poca o escasa atención a la teoría jurídica de la interpretación constitucional por cuanto aparentemente la actividad interpretativa está más encaminada al orden público que al privado.

En síntesis, Troncoso (2010) aclara que "la protección de datos personales debe acompasarse atendiendo a la mayor o menor cercanía con otros derechos fundamentales (...) la protección de datos personales no es sólo un derecho autónomo, sino también una garantía de instituto de otros derechos fundamentales (p. 781). La panacea jurídica en un posible conflicto de derechos e intereses será analizar, evaluar, contrastar o ponderar los mismos de acuerdo a la doctrina constitucional que forma parte del ordenamiento jurídico de los estados.
Cabe señalar, en este contexto, que la intimidad como derecho fundamental, puede verse afectada desde distintos ámbitos de la dignidad humana; siendo necesario establecer los principios fundamentales sobres los cuales se debe propender a garantizar el tratamiento de la información y en donde se considera que la intimidad o el interés público se establece como uno de aquellos frente a la posible amenaza que a la dignidad representa.

Por lo tanto, deben existir ciertos criterios en el manejo de la información que viabilicen la regulación y susceptibilidad o no de tratamiento por terceros. Al referir criterios se está hablando de principios fundamentales que se deben considerar en el tratamiento de la información. Se afirman, de modo general, la confianza y la seguridad, incluida la intimidad, privacidad y confidencialidad, como elementos esenciales en el tratamiento de la información de carácter personal, mismos que desde el ámbito constitucional constituyen garantías jurídicas y que por consiguiente regulan la protección de los datos de carácter personal.

Como se había mencionado anteriormente, al ser considerado como un derecho fundamental la protección de la información de carácter personal se debe propender a efectivizar el control y limitación adecuada al libre tratamiento de la información a través de garantías que desde el nivel constitucional lo haga posible. Por consiguiente, constituye una obligación a que el Estado se encuentra, de modo inexcusable, obligado a brindar a las personas; en tanto que correlativamente las normas que efectivicen la intimidad deberá ser regulada entre los sujetos que formamos parte de la sociedad de la información, estableciéndose al efecto las sanciones que corresponden ante la vulneración de este derecho fundamental.

Según las particularidades léxicojurídicas del país de que se trate, puede conceptuarse al habeas data como una acción, una garantía constitucional, un procedimiento 
jurisdiccional de tramite especial y sumarísimo, un proceso constitucional o un recurso protectorio del derecho de autodeterminación informativa o derecho a la protección de los datos personales frente a posibles excesos del poder de registración precisamente de la información de carácter personal (Bazán, 2005, p. 85-139).

Desde esta concepción se desprende la existencia una garantía individual destinada a propender la protección jurídica de los datos personales. Esta garantía como se ha mencionado tiene relación con el "hábeas data" que tiene por objeto proteger el derecho fundamental a la "autodeterminación informativa" y del cual se desprenden los derechos "arco".

De sus siglas, se entiende que configuran el conjunto de derechos que están destinados a proteger el tratamiento de la información personal; $\mathrm{y}$, en suma, garantizan a las personas el ejercicio, control y poder de disposición de sus datos a través del derecho al acceso, rectificación, cancelación y oposición.

Como señala el jurista ecuatoriano Trujillo (2010):

el derecho de reformar o rectificar puede consistir en actualizar 0 agregar datos nuevos que no constan en el archivo por falta de actualización; suprimir o borrar datos que no corresponden a la verdad, o porque son impertinentes al fin para el que se ha elaborado el archivo (...) rectificar datos que no son exactos, aclarar los datos ambiguos, ininteligibles $\quad 0$ incoherentes, bloquear o asegurar los datos sensibles u otros para que no pueden acceder personas no autorizados (...) En la doctrina se atribuye a cada una de estas facultades del titular el nombre de hábeas data.

Es decir, al ser considerada como una garantía constitucional constituye un mecanismo de acceso, rectificación, cancelación y oposición, a través de los denominados "derechos arco". Sobre la base de estas consideraciones, a continuación, se analizará la heterogeneidad normativa sectorial que en Ecuador se ha desarrollado para la protección del derecho fundamental de datos personales.

En suma, se tratará de responder a las interrogantes inicialmente planteadas en virtud de considerar si el marco jurídico es adecuado para la protección del derecho fundamental a la protección de datos personales. Incluso, repensar en adoptar una normativa más global como ya lo ha hecho la Comunidad Europea mediante el Reglamento (UE) 2016/679 del Parlamento Europeo y del Consejo en el cual se destacan, entre otras, temáticas emergentes como la regulación de los datos sensibles y los derechos de acceso, rectificación, cancelación y oposición.

Por otra parte, el tema en cuestión conlleva a realizar una necesaria distinción entre lo público y lo privado de la información personal. Así, aparentemente la dimensión que envuelve la regulación del derecho fundamental a la protección de datos de carácter personal es que verdaderamente no se ha llegado a considerar como algo importante en su derivación como un derecho fundamental. De hecho, en el ámbito público un dato o determinada información puede tener un grado de interés de general, de donde se desprende que el titular sea de modo voluntario o por mandato de la ley puede o debe compartirlo con terceras personas.

Para comprender mejor esta parte, Troncoso (2010) refiere que "existe un conjunto de datos que se encontrarían en una zona de Core y que tienen una muy elevada capacidad para impedir las injerencias" (p. 781). En esta zona se encontrarían los datos de carácter de especialmente protegidos o privados y que pertenecen a la vida íntima del titular de la información y que afecta de algún modo el ejercicio de sus derechos de personalidad. 
Por el contrario, un dato personal tiene el carácter de público por el interés colectivo que conlleva y se encuentra en una fuente accesible al público. Sin embargo, tal como advierte Troncoso (2010):

sigue vigente en este caso el derecho fundamental a la protección de datos personales como limite a los tratamientos de datos personales, aun de aquellos que aisladamente parece de poca importancia, pero que unidos a otros pueden establecer un perfil determinado de las personas (p. 781).

Se precisa entonces que en relación a la distinción entre lo público de lo privado en el tratamiento de la información personal se asume dos garantías constitucionales, a saber: el derecho de acceso a la información y el habeas data respectivamente. En este contexto, Murillo de la Cueva (2010) agrega que:

El verdadero problema es cualitativo $y$ puede venir --aunque no necesariamente-- de la mano del pragmatismo. Es el que surge cuando, en el marco del contraste entre los intereses en juego, se intente saldar el ajuste entre los que asisten a las personas a quienes pertenece la información y los de quienes la quieren tratar en beneficio de estos últimos, llevando a las normas ese desequilibrio o imponiéndolo en su interpretación.

Es claro que el derecho fundamental a la protección de datos personales se efectiviza a través del habeas data, en donde se asume que el titular de la información personal debe conocer y estar informado sobre el tratamiento y finalidad de su información personal en el ámbito público o privado.

Sirva en esta parte como ejemplo lo que dispone la Constitución de la República del Ecuador en cuanto a la garantía jurisdiccional del habeas data. A saber, el Art. 92 señala:

Toda persona, por sus propios derechos o como representante legitimado para el efecto, tendrá derecho a conocer de la existencia y a acceder a los documentos, datos genéticos, bancos o archivos de datos personales e informes que sobre sí misma, o sobre sus bienes, consten en entidades públicas o privadas, en soporte material o electrónico. Asimismo, tendrá derecho a conocer el uso que se haga de ellos, su finalidad, el origen y destino de información personal y el tiempo de vigencia del archivo o banco de datos.

Las personas responsables de los bancos o archivos de datos personales podrán difundir la información archivada con autorización de su titular o de la ley.

La persona titular de los datos podrá solicitar al responsable el acceso sin costo al archivo, así como la actualización de los datos, su rectificación, eliminación o anulación. En el caso de datos sensibles, cuyo archivo deberá estar autorizado por la ley o por la persona titular, se exigirá la adopción de las medidas de seguridad necesarias. Si no se atendiera su solicitud, ésta podrá acudir a la jueza o juez. La persona afectada podrá demandar por los perjuicios ocasionados.

Por lo que se refiere a la libertad de información se regula a través de la garantía de acceso a la información pública y que precisa desarrollar la facultad de requerir a la Administración Pública determinada información que reviste de interés público.

Del mismo modo, se puede tomar como referencia lo dispuesto por la Constitución de Ecuador en cuanto a la garantía jurisdiccional de Acceso a la Información Pública. A saber, el Art. 91 señala:

La acción de acceso a la información pública tendrá por objeto garantizar el acceso a ella cuando ha sido denegada expresa o tácitamente, o cuando la que se ha proporcionado no sea completa o fidedigna. Podrá ser interpuesta incluso si la negativa se sustenta en el carácter secreto, reservado, confidencial o cualquiera 
otra clasificación de la información. El carácter reservado de la información deberá ser declarado con anterioridad a la petición, por autoridad competente y de acuerdo con la ley.

Más aun, ante el carácter público que puede representar los datos personales de los individuos se pueden identificar, además, algunas características esenciales propias del titular de la información que forman parte de su intimidad.

A saber: se menciona primero el carácter innato, es decir que le corresponde a todo hombre por virtud de su propia humanidad o por el solo hecho de ser persona; segundo, es vitalicia ya que permanece arraigada a la naturaleza humana por toda su vida por ende nunca se la pierde; tercero, es inalienable por cuanto no está sujeta al despojo, privación o tratamiento por terceros de manera arbitraria; cuarto, es imprescriptible lo cual relacionado al carácter vitalicio determina que la intimidad no se la pueda perder por el transcurso del tiempo sin embargo de que el titular de la información haya distraído el manejo de sus datos, es decir los haya abandonado; quinto, es extra patrimonial ya que esta fuera del alcance embargable de los bienes del titular de la información y; sexto es absoluta en cuanto se ejerce erga omnes, es decir se ejerce su efecto con relación a toda la sociedad (Ekmekdjian y Pizzolo, 1996, p. 80).

Es innegable la preocupación de la carencia de políticas, tanto públicas como privadas, que tiendan al control, manejo de la información de carácter personal bajo las consideraciones de respeto a la intimidad personal a que se tiene.

En suma, se mantiene la importancia de determinar las características propias del derecho a la protección de datos personales frente al derecho de acceso a la información conforme a la intención que represente el manejo de la información. Para aquello, es necesario reflejar y determinar el carácter público o cierto orden jurídico en el tratamiento de la información que puede de manera excepcional permitir la libre accesibilidad.

Finalmente, dentro de los resultados de esta investigación un aspecto esencial representa el carácter sensible de la información, es decir el de los datos especialmente protegidos

Inicialmente, los debates legislativos - así lo reflejan la mayoría de textos constitucionales analizados - al parecer estuvieron enmarcados en concentrar la protección de datos personales en los derechos de intimidad y privacidad de las personas. Por tanto, surge la necesidad de distinguir el concepto de intimidad y privacidad a fin de contextualizar el sentido de los datos sensibles en el marco del derecho fundamental a la autodeterminación informativa.

En esta discusión respecto a la relevancia jurídica de la intimidad y privacidad se ha llegado a establecer serias diferencias:

Sobre este particular, se ha afirmado que la intimidad es aquel ámbito de la vida de la persona que se sitúa por completo en la interioridad, fuera del alcance de nadie y, por tanto, ajeno a toda exteriorización y relación, mientras que la vida privada es aquella que se desenvuelve a la vista de pocos, o de otra persona y, en una aceptación más amplia, el conjunto de actos que se realizan o piensan para conocimiento de las personas cercanas (Serna, 1994, p. 215).

Se entendería que la intimidad comprende aquel ámbito de la persona que se encuentra alejado de toda posibilidad de conocimiento de terceras personas, es decir está en lo más interno del ser y fuera del alcance de cualquier persona; mientras que la privacidad sería susceptible de ser revelada a determinadas personas.

Sobre esta base, tal como señala Herrán Ortiz (2002), el concepto de datos sensible se acerca al de intimidad considerando que: 
...los datos sensibles no deben equipararse sin más a datos íntimos porque no siempre es así, y porque la intimidad de la información personal dependerá de cada individuo y de cada situación, ya que no es algo que objetivamente y con carácter general deba determinarse legislativamente" (p. 53).

Sin embargo, resulta importante determinar qué datos se pueden considerar como sensibles frente al tratamiento de la información personal a fin de contextualizar su protección tanto constitucional como sectorial. Estos, como expone Bazán (2005) "pueden ser conceptuados como los datos personales que revelan origen racial y étnico, opiniones públicas, convicciones religiosas, filosóficas o morales, afiliación sindical e información referente a la salud o a la vida sexual" (p. 115).

Bajo la consideración de ocasionar un daño más grave a la dignidad humana y al libre desarrollo de la personalidad, Bazán (2005) agrega sobre los datos sensibles que "es necesario advertir que existen datos en apariencia inocuos o irrelevantes que, de modo súbito, se convierten en extremadamente sensibles mediante un sencillo cambio del fin que se persiguiera al momento de su recolección" (p. 115).

Por tanto, al parecer, se requiere de una clasificación o tipología de datos personales. Así, la doctrina refiere que se pueden clasificar en relación con su mayor o menor relación con el concepto de dignidad y al ejercicio de los derechos fundamentales; existen ciertos datos que no podrían soportar la capacidad de injerencia y afectación a la dignidad, intimidad personal y familiar y al libre desarrollo de la personalidad. Éstos últimos tienen el carácter de sensibles o especialmente protegidos. (Troncoso, 2010, p. 781, 782).

Sin duda, en esta actividad de discernimiento, un papel importante conlleva la estimación o el juicio de valor que se haga desde el ámbito judicial o constitucional resolviendo mediante la acción de habeas data o según la concepción que se tenga en cada Estado - a efecto de resolver según los casos concretos.

Se puede señalar entonces que, por mandato constitucional, en el caso de Ecuador, se estima que los datos sensibles tienen el carácter de íntimo y se los provee de medidas de seguridad necesarias, encaminadas a garantizar la reserva de la información personal por lo que el incumplimiento en este caso conducirá a que el titular de la información pueda invocar la violación del derecho fundamental a la autodeterminación informativa.

\section{DISCUSIÓN}

Esta figura jurídica, de singular aplicación, en los estados constitucionales de derechos consiste en el mecanismo de garantía de que dispone el Estado para efectivizar el cumplimiento de los distintos derechos fundamentales que se consagran en el ámbito constitucional. Es decir, constituyen las acciones de carácter constitucional que tienen previsto asegurar el cumplimiento de los derechos y garantías individuales establecidas en la Constitución como norma suprema del Estado.

Por tanto, como señala Gil Rendón (2004), debe entenderse que por garantías constitucionales se conciben "aquellos procedimientos que se utilizan para restaurar el orden constitucional desconocido o violado" (p. 23). Es ya de apreciación generalizada que el problema de los derechos fundamentales no está en determinar su fundamento ni el reconocimiento interno de que los Estados deben formalizar en sus ordenamientos jurídicos.

El principal problema a que se enfrentan los derechos humanos -que se efectivizan desde el ámbito constitucional mediante las denominadas garantías constitucionales- es su 
ejercicio en la práctica social, que es en donde se determina su eficacia. Al respecto, Gil Rendón (2004) agrega que procedimentalmente las garantías constitucionales deben "restituir el estado de cosas anteriores a la violación, y además implica que se desarrollen plenamente los mandatos constitucionales, para ajustar la Constitución a la realidad y para influir y cambiar la realidad" (p. 23).

Por tanto, según expone Castillo Córdova (2003) en general las garantías constituyen: todo mecanismo jurídico dirigido a hacer efectivo el cumplimiento efectivo de toda la Constitución, como un todo unitario en general (garantías constitucionales), o el cumplimiento efectivo sólo de una parte de la misma, precisamente la que recoge los derechos de las personas (garantías de derechos constitucionales) (p. 243).

En suma, siempre en todo estado constitucional de derechos será necesario establecer un sistema de protección y aseguramiento de los derechos fundamentales con el objeto de garantizar la eficacia de los mismos mediante el restablecimiento del orden constitucional alterado.

Entre las denominadas garantías jurisdiccionales -acorde a nuestro objeto de estudio- se encuentra se encuentra la figura del hábeas data que está encaminada a asegurar lo que se ha denominado como derecho a la protección de la información de carácter personal.

Ahora bien, en relación al hábeas data frente a las nuevas tecnologías, hemos referido que la efectividad de las garantías constitucionales se verifica en la práctica a través del establecimiento de procedimientos o mecanismos jurídicos que tiendan a hacer efectivos los derechos que se consagran en la Constitución como norma suprema dentro de los ordenamientos jurídicos de los Estados.

Así, por ejemplo, se mencionan algunas acciones constitucionales, tales como: hábeas corpus, hábeas, data y acción de protección o de amparo, tendientes a garantizar la vigencia de los derechos humanos en un Estado constitucional de derechos.

En el presente estudio la garantía constitucional o jurisdiccional que resulta de nuestro interés es la acción de habeas data, por cuanto está orientada a satisfacer la protección de nuestro objeto de estudio. Esta garantía según menciona Gozaíni (2001):

...protege el derecho a la intimidad; pero al mismo tiempo se afirma que la defensa es de la privacidad, o de la dignidad humana, o el derecho a la información, o bien la tutela del honor, o de la propia imagen o perfil personal, o derecho a la identidad, o simplemente acotado a la autodeterminación informativa" (p. 13).

Es importante mencionar que, de manera preliminar el hábeas data se trata de una acción, garantía que se hace efectivo a través de un procedimiento jurisdiccional de carácter constitucional encaminado a proteger los datos o información de carácter personal frente al tratamiento de que puede ser objeto la información por terceros. Por tanto, Puccinelli (1999) agrega que:

El habeas data o protección de datos personales, establece garantías mínimas de calidad y confiabilidad de los datos nominativos o personales que se recojan; el derecho de las personas a exigir que sus datos personales le sean exhibidos; el derecho a que sean rectificados y el derecho a excluir los datos privados mantenidos sin autorización (p.351).

De esta manera, se configura como acción procesal que dentro del ámbito constitucional protege los derechos de quienes son titulares de la información y que se traduce en la práctica jurídica como el mecanismo idóneo para precautelar la intimidad de las personas respecto al acceso de terceras personas a la información personal; a que en caso de que la información sea errónea se rectifique y que en 
caso de persistir el uso abusivo se cancele el derecho a seguir regulando el tratamiento de la información mediante la oposición que efectúe el titular de los datos.

En consecuencia, podemos aseverar que esta acción constitucional se ha convertido actualmente en el mecanismo más idóneo para combatir los atentados contra la libertad especialmente en el manejo de la información dentro de la sociedad informatizada. Por tanto, el derecho procesal enfrenta una función tutelar que se vuelve compleja en cuanto a garantizar el adecuado tratamiento de los datos personales convertido en un nuevo poder en la sociedad, de difícil control, producto del desarrollo tecnológico experimentado en los últimos tiempos.

Es preciso señalar también que la existencia de bancos de datos, frecuentemente ha llegado a considerarse como atentatorio a la intimidad personal en los casos -sea en el ámbito público o privado- en que los encargados del tratamiento de la información no prevén los mecanismos necesarios de protección.

Como expone Castillo Córdova (2003), a esto se suma:

...la tarea de control de la existencia y funcionamiento de los bancos de datos, no solo debe quedar en manos del poder político, el cual a través de sus normas puede establecer la regulación más eficaz a cumplir por los titulares del poder informático; sino que debe recaer también en los particulares (371).

Al haber fijado la problemática de la garantía de los datos personales en cuanto al manejo de la información de los titulares de datos sensibles, la garantía constitucional más idónea para efectivizar su cumplimiento es la acción de hábeas data por cuanto está encaminada a proteger todos los derechos que se desprenden del manejo de los datos o información, es decir se convierte en el procedimiento constitucional efectivo para garantizar la actuación inmediata del órgano judicial correspondiente en los casos de violación del derecho a la intimidad que resultan como consecuencia del manejo de bancos de datos tanto públicos como privados.

\section{CONCLUSIÓN}

En el caso de Ecuador, así como la mayoría de países de Latinoamérica, puede decirse que el habeas data ha tenido más desarrollo que el derecho fundamental a la protección de datos personales propiamente dicho. En este reconocimiento debe destacarse que uno de los méritos más altos está el haberse atribuido autonomía al derecho fundamental que se desprende del tratamiento de la información personal. Le sigue en importancia, la ampliación y desarrollo de la garantía constitucional del habeas data al habérsele incorporado más facultades procesales y garantistas sobre este derecho fundamental.

Ahora bien, el hábeas data como un mecanismo de garantía procesal frente a las nuevas tecnologías representa uno de los principales avances en materia constitucional. No se trata que en el derecho procesal constitucional a través del habeas data simplemente se facilite el acceso a la información personal sino más bien de controlar el uso ilícito que terceras personas responsables de tratamiento- hagan de los datos personales. Consecuente, el escenario es mucho más amplio, se pretende que el habeas data sea considerado como un mecanismo de protección del instituto de garantía que constituye el derecho fundamental a la protección de datos personales.

\section{REFERENCIAS}

Bazán, V. (2005). El habeas data y el derecho a la autodeterminación informativa en perspectiva de Derecho Comparado, Revista Semestral del Centro de Estudios Constitucionales: Universidad de Talca 
Castillo Córdova, L. (2003). Elementos de una teoría general de los derechos constitucionales, Lima-Perú, ARA Editores

Davara, M. (2005). Anuario de Derecho de las Tecnologías de la Información y las Telecomunicaciones (TIC) Trabajos doctrinales especializados, boletines de actualidad, reseñas de interés jurídico, glosario de términos, preguntas más frecuentes y otras informaciones de interés. Madrid, España: Fundación Airtel

Ekmekdjian, M. Á. y Pizzolo, C. (1996). Habeas data. El derecho a la intimidad frente a la revolución informática, Buenos Aires, Depalma

Gozaíni, O. (2010). Derecho procesal constitucional: Hábeas Data-Protección de datos personales, Buenos Aires, RubinzalCulzoni Editores

Gil Rendón, R. (2004). Derecho procesal constitucional. México, Fundap.

Herrán Ortiz, A. (2002). El derecho a la intimidad en la nueva ley orgánica de protección de datos personales, Madrid, Dykinson.
Murillo de la Cueva P. y Piñar J. L. (2011). El derecho a la autodeterminación informativa (Madrid-México: Fontamara S.A.

Murillo de la Cueva, Pablo. (2010), La protección de los datos de carácter personal en el horizonte de 2010, Navarra, Anuario Facultad de Derecho Universidad de Alcalá.

Pérez Luño, A. (1994). Manual de Informática y Derecho, Madrid, Editorial Ariel S.A.

Puccinelli, O. (1999). El Habeas Data en Indoiberoamérica, Santa Fe de BogotáColombia, Editorial Temis S.A.

Serna, P. (1994). Derechos fundamentales: el mito de los conflictos. Reflexiones teóricas a partir de un supuesto jurisprudencial sobre intimidad e información, Pamplona.

Troncoso A. (2010). La Protección de Datos Personales: En busca del equilibrio. Valencia: Tirant lo Blanch.

Trujillo, J. C. (2010), Las Garantías Jurisdiccionales, Base de datos: Vlex.com.ec: https://app.vlex.com/\#WW/vid/515951 146/graphical_version. 